\title{
Drought Stress Influences Gas-exchange Responses of Papaya Leaves to Rapid Changes in Irradiance
}

\author{
Hiphil S. Clemente and Thomas E. Marler \\ College of Agriculture and Life Sciences, University of Guam, Mangilao, Guam 96923 \\ Additional index words. Carica papaya, cloud cover, net $\mathrm{CO}_{2}$ assimilation, photosynthetic photon flux, stomatal conduc- \\ tance, tropical, water use efficiency
}

\begin{abstract}
Field-grown 'Red Lady' papaya (Carica papaya L.) plants were used to measure foliar gas-exchange responses to rapid changes in irradiance levels to determine if papaya stomata are able to track simulated sun-to-cloud cover transitions. Natural sunlight and neutral shade cloth placed over the leaf were used to provide high photosynthetic photon flux (PPF) of about $2000 \mu \mathrm{mol} \cdot \mathrm{m}^{-2} \cdot \mathrm{s}^{-1}$ until leaves reached steady state within the cuvette, followed by three minutes with low PPF of about $325 \mu \mathrm{mol} \cdot \mathrm{m}^{-2} \cdot \mathrm{s}^{-1}$, and a return to PPF of about $2000 \mu \mathrm{mol} \cdot \mathrm{m}^{-2} \cdot \mathrm{s}^{-1}$. Net $\mathrm{CO}_{2}$ assimilation (A) declined from an initial $20 \mu \mathrm{mol} \cdot \mathrm{m}^{-2} \cdot \mathrm{s}^{-1}$ to about $9 \mu \mathrm{mol} \cdot \mathrm{m}^{-2} \cdot \mathrm{s}^{-1}$ within 20 seconds of initiating low PPF, and remained fairly stable for the duration of the three minutes of low PPF. Stomatal conductance $\left(g_{s}\right)$ declined within 60 seconds of initiating low PPF, from 385 to about $340 \mu \mathrm{mol} \cdot \mathrm{m}^{-2} \cdot \mathrm{s}^{-1}$ during the three minutes duration of low PPF. Following the return to high PPF, A rapidly increased to about $18 \mu \mathrm{mol} \cdot \mathrm{m}^{-2} \cdot \mathrm{s}^{-1}$, then gradually increased to the original value. After a lag of about 1 minute following the return to high PPF, $g_{s}$ began to increase and returned to the original value after three minutes. Container-grown 'Tainung \#1' papaya plants were used in a second study to determine the influence of mild drought stress on gas-exchange responses to rapid irradiance transitions. For drought-stressed plants, $g_{\mathrm{s}}$ declined to a greater magnitude following the high-to-low PPF transition, and $\mathrm{g}_{\mathrm{s}}$ and A recovered more slowly following the transition from low-to-high PPF than for well-watered plants. Water use efficiency declined to a minimum immediately following the high-to-low PPF transition for both sets of plants, but recovered more rapidly for drought-stressed plants. These results indicate that papaya stomata are able to track rapid changes in irradiance, and mild drought stress enhances the tracking response.
\end{abstract}

Determining the dependence of stomatal conductance and photosynthesis on irradiance levels are useful measurements in ecophysiology research and in modeling plant growth and development (Schaffer and Andersen, 1994). The response of photosynthesis to light for $\mathrm{C}_{3}$ plants, for example, typically exhibits a linear phase where light is the most limiting factor, a curvilinear phase as light becomes of decreasing importance as a limiting factor, and a plateau above which further increases in irradiance cause no further increase in photosynthesis. Data for determining these light response curves are obtained under steady state conditions, where leaves are provided a time interval long enough to adjust to each new level of irradiance.

Most plants experience continuous fluctuations in light under natural conditions rather than long periods of uniform irradiance. The most obvious light dynamics are those experienced by understory plants or the innermost leaves in a large canopy, where low irradiance is periodically punctuated by high irradiance in the form of sunflecks lasting from seconds to minutes (Pearcy, 1988). Even individuals of pioneer and other species growing in open habitats experience high and low light periods in the order of several minutes each due to broken cumulus cloud cover (Knapp and Smith, 1990). In Guam, only 18 days per year are classified as clear (National Oceanic and Atmospheric Administration, 1993). The remaining days are cloudy or partly cloudy, mostly due to broken cumulus cloud cover. These weather conditions are typical for lowland tropical environments (Tseng and Muniappan, 1986). Gas-exchange of plants in these locations may rarely obtain steady state conditions with respect to irradiance, but may alternatively be in a constant state of responding to an integration of instantaneous

Received for publication 10 May 1995. Accepted for publication 10 July 1995. This research was supported by the Univ. of Guam Graduate Council and Office of the Graduate School and Research, and the U.S. Dept. of Agriculture under CSRS special grant agreement no. 93-34135-8828, managed by the Pacific Basin Administrative Group (PBAG). The cost of publishing this paper was defrayed in part by the payment of page charges. Under postal regulations, this paper therefore must be hereby marked advertisement solely to indicate this fact. irradiance with the most recent abrupt irradiance transitions.

With rapid reductions in irradiance, photosynthesis declines due to rapid biochemical adjustments. With the return to high irradiance, and in the absence of any stomatal limitation, photosynthesis quickly increases. The response of $g_{s}$ to irradiance transitions is more variable among species. Plants have been classified into two broad groups with respect to stomatal responses to irradiance transitions (Knapp and Smith, 1990). Some species exhibit partial stomatal closure with the onset of reduced irradiance, lagging slightly behind the decline in photosynthesis. With the return to high irradiance, $\mathrm{g}_{\mathrm{s}}$ increases and lags behind the increase in photosynthesis. This lag in $g_{\mathrm{s}}$ recovery may limit photosynthesis for a short time following the return to high irradiance. In contrast, $g_{s}$ of other species responds little to abrupt changes in irradiance. In general, herbaceous species growing in the absence of overstory shade tend to be able to track short-term changes in irradiance with respect to $g_{s}$, and woody species tend to have nonresponsive stomata to these short-term changes (Knapp and Smith, 1989, 1990). Furthermore, water stress may alter the gas-exchange response to rapid changes in irradiance of a species (Davies and Kozlowski, 1975; Knapp and Smith, 1989).

Papaya is widely grown under cultivation and in naturalized habitats in the lowland tropics. It is a unique species with respect to the two broad group classifications of Knapp and Smith (1990). Although papaya is herbaceous, its stature is not that of a typical herbaceous plant. Papaya plants may reach heights of $9 \mathrm{~m}$, and are thus described as giant herbs (Malo and Campbell, 1986).

The objectives of this study were to determine the gas-exchange responses of papaya leaves to simulated sun-cloud cover transitions to classify the species with respect to stomatal tracking. Since many lowland tropical islands have distinct dry seasons that accompany the partly cloudy sky conditions, we further determined the influence of mild drought stress on these gas-exchange responses. These data may contribute to a more thorough understanding of the influence of natural heterogeneous irradiance conditions common in lowland tropical environments on the 
integrated daily carbon gain and water loss of papaya plants.

\section{Materials and Methods}

Field study. Ten 'Red Lady' papaya plants about $175 \mathrm{~cm}$ in height were used to determine gas-exchange responses to suncloud transitions. The plants were grown under standard management procedures (Malo and Campbell, 1986), and received $13 \mathrm{~mm}$ of water at least every other day via rainfall or microsprinkler irrigation. Soil type was classified as Clayey, gibbsitic, nonacid, isohyperthermic Lithic Ustorthents.

Gas-exchange responses to natural sun-cloud transitions were measured on numerous days during April and May 1994. Photosynthetic photon flux (PPF) and duration of cloud cover and full sun exposure were highly variable due to variability in cumulus cloud size and distance between clouds. This stochastic natural condition negated the possibility of combining data from several sun-cloud transitions.

To standardize the PPF and duration of the light-limiting phase, measurements were made during a period of atypical, clear days in June 1994. Mid-morning PPF was about $2000 \mu \mathrm{mol} \cdot \mathrm{m}^{-2} \cdot \mathrm{s}^{-1}$ on these days. Sun-to-cloud cover transition was simulated with neutral density shade cloth placed over the laminae, and cloud cover-to-sun transition was simulated by removing the shade cloth after a period of $3 \mathrm{~min}$. PPF during the high irradiance period ranged from 1940 to $2050 \mu \mathrm{mol} \cdot \mathrm{m}^{-2} \cdot \mathrm{s}^{-1}$, and during the $3 \mathrm{~min}$ low irradiance period from 310 to $325 \mu \mathrm{mol} \cdot \mathrm{m}^{-2} \cdot \mathrm{s}^{-1}$. These values were selected based on the range of measured PPF and duration of low irradiance during our initial attempts to measure gas-exchange under natural cloud cover. This procedure allowed repeated measurements with designated high-low-high irradiance transitions.

Gas-exchange was measured on $2.5 \mathrm{~cm}^{2}$ of leaf surface using an open gas-exchange system (CIRAS-1; PP Systems, Stotfield, Hitchin, Herts, U.K.). Measurements were restricted to the most recently expanded leaves. Gas-exchange of leaves was measured under sunlight to provide initial steady-state values. Mean initial values were determined from three records on each plant. With placement of the shade cloth designated as time zero, leaf gasexchange was recorded at 20 -sec intervals. After removing the shade cloth, recovery of gas-exchange characteristics was measured until characteristics returned to the initial steady state values. Once the length of time was determined for this entire process (about $8 \mathrm{~min}$ ) several measurements were made with the cuvette in place for 10 min under steady state PPF to verify that leaf gasexchange did not respond to being loaded in the cuvette.

Five measurements were made during mid-morning on 3 and 4 June, for a total of 10 replications. Conditions during the measurement periods were $345-355 \mu \mathrm{l} \cdot$ liter $^{-1}$ ambient $\mathrm{CO}_{2}, 31-32 \mathrm{C}$, and $1.7-2.1 \mathrm{kPa}$ vapor pressure deficit (VPD). Stomatal conductance, $\left(\mathrm{g}_{\mathrm{s}}\right)$ net $\mathrm{CO}_{2}$ assimilation (A), and water use efficiency (WUE, defined as $\mathrm{A} /$ transpiration) data were plotted as mean \pm standard error.

Container study. Seeds of 'Tainung \#1' papaya were planted in 0.625-liter containers on 1 Aug. 1994, and grown under 75\% sunlight transmission until 17 Sept. 1994. At this time, the plants were transplanted into 2.6-liter containers and transferred to full sunlight. Growing medium was Sunshine Mix 4 (Sun Gro Horticulture, Bellevue, Wash.). The plants received daily watering and weekly fertilization of $150 \mathrm{ml}$ per plant of a complete nutrient solution (Excel; Grace-Sierra, Milpitas, Calif.). The concentration of the solution was based on $7.5 \mathrm{~mm}$ nitrogen.

Daily water use of about $175 \mathrm{ml}$ was determined by loss in mass on several days in late November. A slow drying cycle was begun on half (eight) of the plants beginning 30 Nov. by watering with $100 \mathrm{ml}$ per plant each morning. Net $\mathrm{CO}_{2}$ assimilation of droughtstressed plants had declined to and stabilized at about $70 \%$ of that of well-watered plants by $10 \mathrm{Dec}$. Matric potential of the growing medium was $-31 \pm 4 \mathrm{kPa}$ at this time.

Four plants from each treatment were transferred to a laboratory for gas-exchange measurements. The cuvette from the gas-exchange system previously described was positioned to obtain a PPF of 1600 $\mu \mathrm{mol} \cdot \mathrm{m}^{-2} \cdot \mathrm{s}^{-1}$ from a $1500 \mathrm{~W}$ quartz halogen lamp positioned above a $2.5 \mathrm{~cm}$ thermal-filtering water bath. A leaf was inserted into the cuvette and allowed to reach steady state conditions. Gas-exchange measurements and administration of the low irradiance period were the same as for the field study. Placement of the shade cloth over the leaf provided a PPF of $250 \mu \mathrm{mol} \cdot \mathrm{m}^{-2} \cdot \mathrm{s}^{-1}$. Recovery of gas-exchange following the return to high irradiance was measured for a $3 \mathrm{~min}$ period.

Measurements were made on four replications each of wellwatered and drought-stressed plants. The process was repeated on $11 \mathrm{Dec}$. on four more replications per treatment for a total of eight replications. Conditions during the measurement periods were 345 $-350 \mu \mathrm{l} \cdot$ liter $^{-1}$ ambient $\mathrm{CO}_{2}, 30-31 \mathrm{C}$, and 1.2-1.4 kPa VPD.

Gas-exchange characteristics were standardized by setting the initial steady-state measurements as 100 . This provided an opportunity to more easily compare the relative stomatal tracking response for the two treatments. Data were plotted as mean \pm standard error for each treatment.

\section{Results}

Field study. Assimilation of papaya leaves declined to less than $50 \%$ of the initial high irradiance value within $20 \mathrm{sec}$ of the highto-low irradiance transition (Fig. 1). Within $60 \mathrm{sec}$ of the high-tolow irradiance transition $\mathrm{g}_{\mathrm{s}}$ began a gradual decline that continued for the duration of the low irradiance period. Stomatal conductance began recovering one minute following the low-high irradiance transition, and returned to the initial steady state value after 3 min. This slow recovery of $g_{s}$ was limiting to the recovery of $A$, since almost 2 min following the low-to-high irradiance transition were required for $\mathrm{A}$ to return to the initial value.

WUE declined to a minimum immediately after the high-to-low

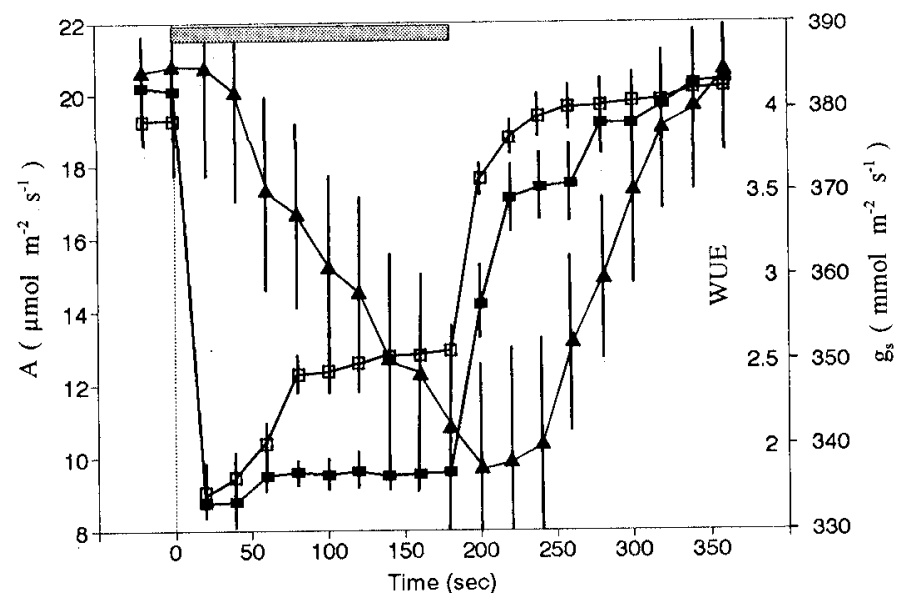

Fig. 1. Response of net $\mathrm{CO}_{2}$ assimilation $(\mathrm{A}, \boldsymbol{\square})$, stomatal conductance $\left(\mathrm{g}_{\mathrm{s}}, \mathbf{\Delta}\right)$ and water use efficiency ( WUE, $\square$ ) determined as A/transpiration of 'Red Lady' papaya leaves to variations in sunlight. The shaded bar indicates $3 \mathrm{~min}$ of low irradiance (photosynthetic photon flux $=310-325 \mu \mathrm{mol} \cdot \mathrm{m}^{-2} \cdot \mathrm{s}^{-1}$ ). Time intervals without the shaded bar indicate full sunlight (photosynthetic photon flux $=1940$ to $\left.2050 \mu \mathrm{mol} \cdot \mathrm{m}^{-2} \cdot \mathrm{s}^{-1}\right)$. Symbols are mean $\pm \mathrm{SE} . \mathrm{n}=30$ for initial data point, $\mathrm{n}=$ 10 for remaining data points. 
irradiance transition, since A declined immediately and $\mathrm{g}_{\mathrm{s}}$ had not yet begun to decline (Fig. 1). However, WUE increased during the low irradiance period as $g_{s}$ gradually declined, and recovered to almost $70 \%$ of the original value by the end of the 3 min period of low PPF. Stomatal limitation of water loss caused WUE to return to the initial steady state value within $40 \mathrm{sec}$ after the low-to-high irradiance transition.

Container study. The relative responses of gas-exchange characteristics to light transitions of well-watered container-grown plants were similar to those of the field-grown plants (Fig. 2A), but absolute values of $\mathrm{A}, \mathrm{g}_{\mathrm{s}}$, and WUE were slightly lower. By $20 \mathrm{sec}$ following the high-to-low irradiance transition, A had dropped to $45 \%$ of the steady state value. However, 1 min of low irradiance was required for $\mathrm{g}_{\mathrm{s}}$ to begin a slow decline, and it was about $90 \%$ of the steady state value after $3 \mathrm{~min}$. A slow recovery of $g_{s}$ began within 1 min of returning to high irradiance, but full recovery was not achieved until after 2 min of high irradiance. As a result, A did not return to original values until about 2 min following the lowto-high irradiance transition. Water use efficiency returned to original values within $40 \mathrm{sec}$ following the low-to-high irradiance transition due to the slow recovery of $\mathrm{g}_{\mathrm{s}}$.

Drought-stressed papaya plants exhibited a more pronounced stomatal tracking response to the abrupt transition from high to low irradiance (Fig. 2B). By $40 \mathrm{sec}$ following the high-low irradiance transition $\mathrm{g}_{\mathrm{s}}$ began declining until it was about $80 \%$ of the initial

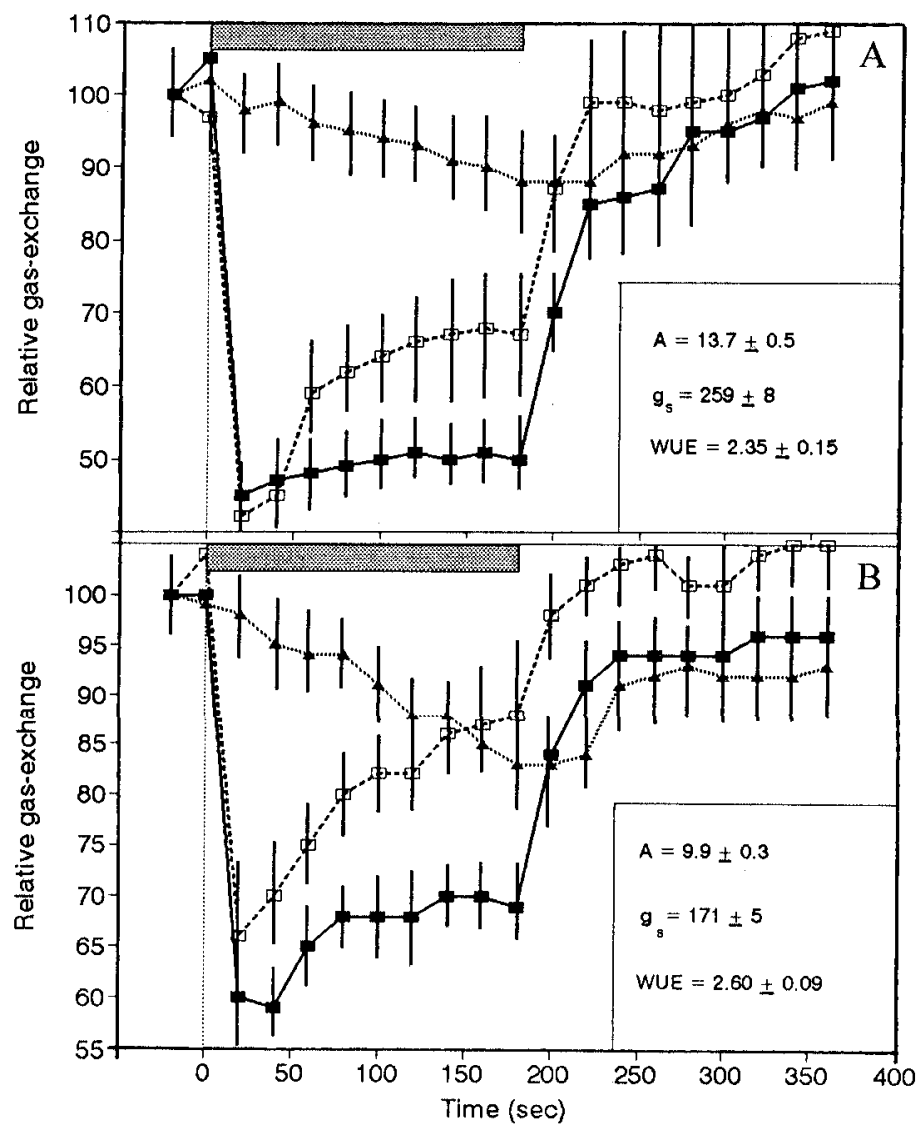

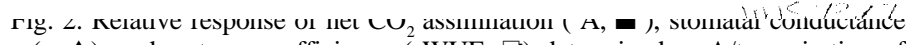
$\left(g_{s}, \mathbf{A}\right)$, and water use efficiency ( WUE, $\left.\square\right)$ determined as A/transpiration of 'Tainung \#1' papaya leaves on $(\mathbf{A})$ well-watered and $(\mathbf{B})$ drought-stressed plants to variations in irradiance. The shaded bar indicates 3 min of low irradiance (photosynthetic photon flux $=250 \mu \mathrm{mol} \cdot \mathrm{m}^{-2} \cdot \mathrm{s}^{-1}$ ). Time intervals without the shaded bar indicate high irradiance (photosynthetic photon flux $=1600 \mu \mathrm{mol} \cdot \mathrm{m}^{-2} \cdot \mathrm{s}^{-1}$ ). Symbols are mean \pm SE. $n=24$ for initial data point, $n=8$ for remaining data points. Insert: initial steady state values. steady state value. Although recovery of $g_{s}$ began shortly after the low-high irradiance transition, full recovery had not been achieved by $3 \mathrm{~min}$.

Under steady state conditions, WUE of drought-stressed plants was $10 \%$ higher than that of well-watered plants. Water use efficiency of drought-stressed plants also declined to a minimum immediately after the high-to-low irradiance transition, but began recovering within $40 \mathrm{sec}$ due to the earlier decline in $\mathrm{g}_{\mathrm{s}}$ (Fig. 2B). Moreover, WUE returned to the initial steady state value by $20 \mathrm{sec}$ after the low-to-high irradiance transition.

\section{Discussion}

Strong trade winds that cause large cumulus clouds to move quickly across the sun's pathway are common in the lowland tropics of the Pacific. These occurrences produce numerous changes in irradiance levels throughout the day. Papaya leaves react to these transitions with a tracking response by stomata, whereby $g_{s}$ declined in response to a rapid decline in irradiance. Tracking of $g_{s}$ was not of the same speed or magnitude as that of A, but it did limit water loss fairly quickly under simulated cloud cover.

This tracking response provided an increase in WUE during the periods of low irradiance. Moreover, WUE returned to maximum almost immediately after the return to full sun. These results indicate that the rapid response of $\mathrm{g}_{\mathrm{s}}$ would allow papaya plants to maximize WUE throughout the day.

A consequence of maximizing WUE by minimizing water loss during episodes of low irradiance is that carbon gain is not maximized. Water conservation at the expense of carbon acquisition is probably adaptive in water-limited habitats (Cowan, 1982). The benefits of this response depend on soil water status and the number of sun-cloud transitions throughout the photoperiod. In the presence of a large number of sun-cloud transitions, a slower depletion of soil moisture between irrigation or rainfall events by stomatal tracking would lower the possibility of prolonged plant moisture stress, at least in relation to a nontracking species with similar steady state transpiration capacity.

Plants experiencing little water stress may maintain higher $g_{s}$ during cloud cover than plants under more severe water stress (Knapp and Smith, 1988). This was evident with the papaya plants, as the relative reduction in $\mathrm{g}_{\mathrm{s}}$ during simulated cloud cover was much greater for drought-stressed plants than for well-watered plants. Knapp and Smith (1990) reported that some species even switch from a nontracking stomatal behavior under well-watered conditions to a tracking stomatal behavior under conditions of water stress.

Regardless of the degree of edaphic water stress, plants with large leaves may maintain more favorable water balance on days with broken cloud cover than during cloudless days (Knapp and Smith, 1989). Total integrated $\mathrm{CO}_{2}$ uptake may be much greater for these species under intermittent sun/shade periods than under continuous sun periods, due to increased leaf temperature and eventual negatively impacted plant water status under continuous sun. We did not measure integrated $\mathrm{CO}_{2}$ uptake during broken cloud cover vs. continuous sunny conditions. However, papaya plants bear large leaves about $1 \mathrm{~m}$ in diameter (Malo and Campbell, 1986), and midday stomatal closure occurs in well-watered papaya plants during clear days on Guam (T.E. Marler, unpublished data). These characteristics indicate that papaya may be among those species that would exhibit a greater daily carbon gain on days with intermittent cloud cover than on clear days, despite the stomatal limitation of A for a short time following each cloud-to-sun transition.

In summary, papaya leaves tracked rapid transitions in incident 
irradiance with partial stomatal closure when A was limited by low irradiance. This response maximized WUE during and shortly after each simulated cloud cover interval. Mild drought stress enhanced the tracking response of papaya leaves.

\section{Literature Cited}

Cowan, I.R. 1982. Regulation of water use in relation to carbon gain in higher plants, p. 589-613. In: O.L. Lange, P.S. Nobel, C.B. Osmond, and H. Ziegler (eds.). Physiological plant ecology. vol. 12B. SpringerVerlag, Berlin.

Davies, W.J. and T.T. Kozlowski. 1975. Stomatal responses to changes in light intensity as influenced by plant water stress. For. Sci. 21:129-133.

Knapp, A.K. and W.K. Smith. 1988. Effect of water stress on stomatal and photosynthetic responses in subalpine plants to cloud patterns. Amer. J. Bot. 75:851-858.
Knapp, A.K. and W.K. Smith. 1989. Influence of growth form and water relations on stomatal and photosynthetic responses to variable sunlight in subalpine plants. Ecology 70:1069-1082.

Knapp, A.K. and W.K. Smith. 1990. Stomatal and photosynthetic responses to variable sunlight. Physiol. Plant 78:160-165.

Malo, S.E. and C.W. Campbell. 1986. The papaya. Univ. of Florida Cooperative Extension ServiceFruit Crops Fact SheetFC-11. Gainesville, Fla.

National Oceanic and Atmospheric Administration. 1993. Local climatological data - Pacific Islands. (C55.286/6-39:993/13).

Pearcy, R.W. 1988. Photosynthetic utilization of lightflecks by understory plants. Austral. J. Plant Physiol. 15:223-238.

Schaffer, B. and P.C. Andersen. 1994. (eds.). Handbook of environmental physiology of fruit crops. vol. 1. Temperate crops. CRC Press, Boca Raton, Fla.

Tseng, C.T. and R. Muniappan. 1986. Agroclimatic atlas of Guam. Univ. of Guam Agr. Expt. Sta. Manual. Mangilao, Guam. 